\title{
Pararotor Dynamics: Center of Mass Displacement from the Blade Plane-Analytical Approach
}

\author{
Joaquín Piechocki* and Vicente Nadal Mora ${ }^{\dagger}$ \\ Universidad Nacional de La Plata, 1900 Buenos Aires, Argentina \\ and \\ Ángel Sanz Andrés ${ }^{\ddagger}$ \\ Universidad Politécnica de Madrid, E-28040 Madrid, Spain
}

DOI: $10.2514 / 1 . C 032378$

\begin{abstract}
The pararotor is a biology-inspired decelerator device based on the autorotation of a rotary wing whose main purpose is to guide a load descent into a certain atmosphere. This paper focuses on a practical approach to the general dynamic stability of a pararotor whose center of mass is displaced from the blade plane. The analytical study departs from the motion equations of pararotor flight, considering the center of mass displacement from the blade plane, studied over a number of simplifying hypotheses that allows determining the most important influences to flight behavior near equilibrium. Two practical indexes are developed to characterize the stability of a pararotor in terms of geometry, inertia, and the aerodynamic characteristics of the device. Based on these two parameters, a stability diagram can be defined upon which stability regions can be identified. It was concluded that the ability to reach stability conditions depends mainly on a limited number of parameters associated with the pararotor configuration: the relationship between moments of inertia, the position of the blades, the planform shape (associated with the blade aerodynamic coefficients and blade area), and the vertical distance between the center of mass and the blade plane. These parameters can be evaluated by computing practical indexes to determine stability behavior.
\end{abstract}

\section{Nomenclature}

$\begin{array}{ll}C_{D} & = \\ C_{M} & = \\ C_{M i} & = \\ C_{L \alpha} & = \\ D_{i j} & = \\ F & \\ I_{a} & = \\ I_{i} & = \\ k & \\ k_{21} & = \\ k_{31} & = \\ L_{i j} & = \\ M_{i} & = \\ N_{e}^{\prime} & = \\ P_{\mathrm{amb}} & = \\ r_{i j} & = \\ S & = \\ T_{\mathrm{amb}} & =\end{array}$

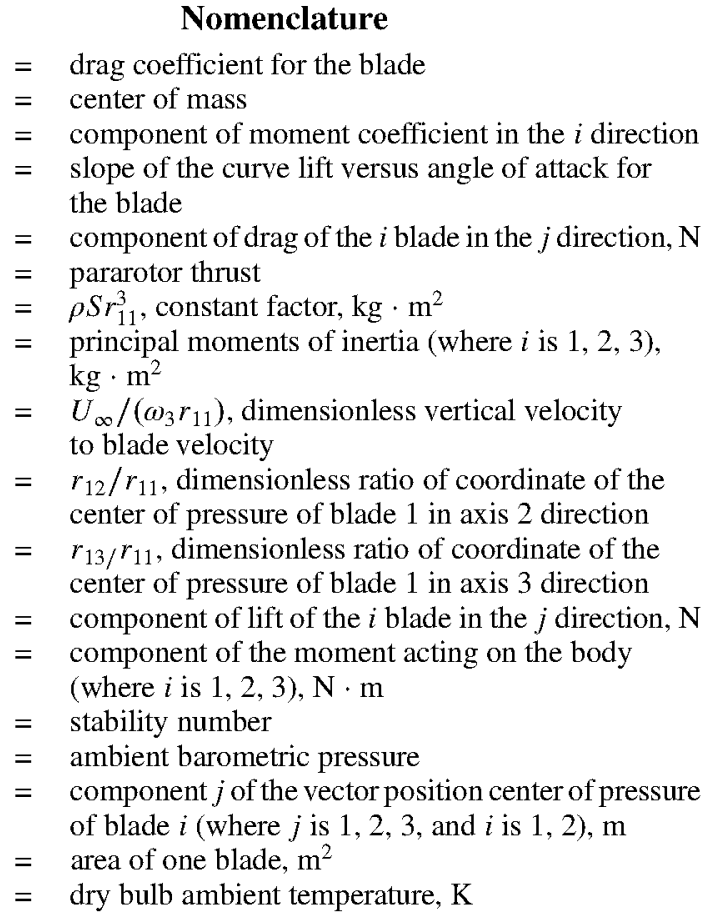

Received 9 April 2013; revision received 29 August 2013; accepted for publication 29 August 2013; published online 5 February 2014. Copyright $(C$ 2013 by The authors. Published by the American Institute of Aeronautics and Astronautics, Inc., with permission. Copies of this paper may be made for personal or internal use, on condition that the copier pay the $\$ 10.00$ per-copy fee to the Copyright Clearance Center, Inc., 222 Rosewood Drive, Danvers, MA 01923; include the code 1542-3868/14 and $\$ 10.00$ in correspondence with the CCC.

*Teacher, Departamento de Aeronáutica, Facultad de Ingeniería, Calle 116 Entre 47 y 48.

${ }^{\dagger}$ Professor, Departamento de Aeronáutica, Facultad de Ingeniería, Calle 116 Entre 47 y 48 . Senior member AIAA.

"Professor, Instituto Universitario de Microgravedad "Ignacio Da Riva," Escuela Técnica Superior de Ingenieros Aeronáuticos, Plaza Cardenal Cisneros, 3 .

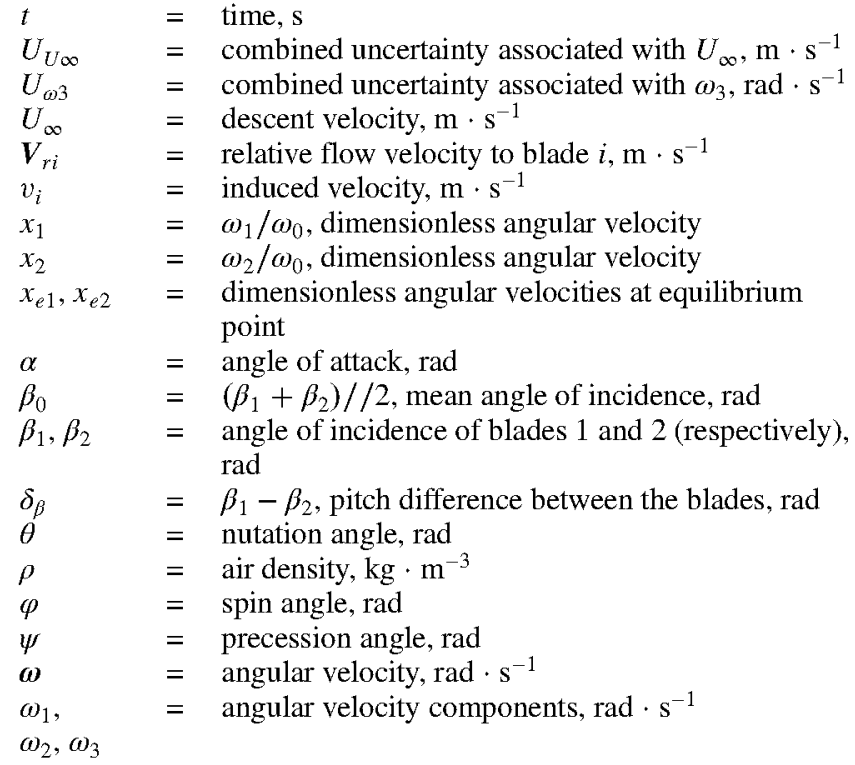

\section{Introduction}

The pararotor is a biology-inspired rotary decelerator whose main objective for practical use is to aerodynamically guide a load descending into a certain atmosphere or to perform measurements during descent. The possible applications of such a probe could be, for instance, the measurement of the atmospheric conditions around airports for aviation operations support or the exploration of planetary atmospheres.

Presently, there are many devices that perform tasks such as measuring winds, emissions, and atmospheric parameters for aviation support. Each of them has its own advantages and drawbacks. The pararotor could contribute to this set of systems with its own capabilities. The pararotor flies in the so-called autorotation regime, where aerodynamic forces are generated, taking energy out from the fluid flow. These forces produce a fast rotation motion, which induces a large amount of lift on the rotating blades, seen as a net aerodynamic drag acting on the body. The "autorotation" name is used here in the sense that the net torque is zero. 
Although the autorotation regime is widely known for applications in helicopters, the problem studied here is different due to the small aspect ratio of the pararotor blades, while in helicopter rotors, the aspect ratio is very large. In pararotors, the aspect ratio is about 1, and so the aerodynamic approach developed for helicopters cannot be applied. The small blade aspect ratio allows them to be folded over the pararotor body, in case that could be a design requirement.

Pararotors can be defined as falling bodies with control capabilities for descent a load. Among the studies performed concerning the deceleration and control of falling bodies, there are those carried out by Shpund and Levin [1-4] in the area of rotating parachutes, while Karlsen et al. [5] worked on winged bodies for submunition applications. The latter reported the following advantages of the pararotor over the parachute: lower sensitivity to lateral winds, no parachute deployment problems, lower precession movements, and higher falling velocity.

The flight of samara wings has similarities with that of pararotors. Rosen and Seter [6,7] have studied numerically the influence of different parameters on samara wing flight stability. Crimi [8] has studied a rotating body with only one wing for submunition applications, searching for a body that performed periodic movements. However, in these papers, there is no information about the behavior of a body with rotating wings like that presented here, and to the authors' knowledge, there is no other closely related information in the literature.

Previous work has been done to model the stability of a pararotor, mainly by Nadal Mora, Sanz, and Piechocki [9-16]. They conducted investigations that present the stability behavior of pararotors whose blades were aligned with the center of mass of the whole device. They developed an analytical model that predicts the dynamic behavior under different device configurations.

Pararotor decelerator systems were compared with other methods of entry probe atmospheric deceleration by different authors $[17,-20]$ and were determined to show significant potential for application to mission concepts requiring controlled descent, low-velocity landing, and atmospheric research capability on planet exploration.

The main objective of the current work is to help depict the stability behavior of a pararotor, considering different design parameters. Better understanding the behavior of the pararotor could make it possible to know how to manage the system parameters to attain a desired functional mission. This study contributes to the development of tools to design and control pararotors.

An analytical approach will be used to study the dynamic behavior, which will account for the main effect of parameters design, particularly the distance between the blade plane and the center of mass of the pararotor. This model will take into account the flight regimes near the equilibrium point.

The main conclusion is that a number of parameters that affect the equilibrium attitude and dynamics of pararotors can be analyzed by expanding the aerodynamic torque involved in the Euler equations of the system for flight conditions close to the equilibrium.

When considering the different configurations and physical parameters of the pararotor, the aspect most relevant to dynamic stability and attitude is the relationship between the device principal moments of inertia. In practical terms, only some configurations appear to be stable. The vertical distance between the blade plane and the center of mass affects the equilibrium position and the stability of the system, making it closer to or further from its dynamic stability limits.

The work performed and reported in this paper is structured as follows. In Sec. II, the motion equations for a cylindrical body with two identical blades are developed, and the stability analysis is presented. In Sec. III, some experimental results are included to support the assumptions considered. In Sec. IV, the results of the work are outlined. In Sec. V, a discussion is presented. And in Sec. VI, conclusions are drawn.

\section{Materials and Methods}

The approach chosen to study the dynamic behavior of pararotors was to establish an analytical model of this device based on the Euler equations and to identify the most important terms. Althought linear accelerations can be relevant in part of the flight envelope of pararotor (namely the starting phase), once the steady motion is stablished, the descent speed remains constant. We consider that the descent speed is not significantly perturbed by the small deviations of pararotor attitude, as it was observed in the experiments performed. Therefore, Newton equations of forces are decoupled from the rotational dynamics and not considered because the problem analysis is focused on an equilibrium solution where a rotation limit speed is held relatively constant, and translational accelerations are zero. To this aim, certain simplifying assumptions were introduced. The motion equations were linearized and uncoupled to obtain the constant matrixes of a linear system, dependent on the parameters of the pararotor configuration. The design parameters, because the control is fixed, determine the trim state previous to disturbances and therefore the stability matrix. A classical eigenvalue study was performed to analyze the stability behavior, and equilibrium points were also determined.

A schematic representation of the geometry of a pararotor is shown in Fig. 1, introducing the center of mass position and center of pressure for each blade, expressed in the body axis; $r_{i j}$ is then defined as the coordinate of the center of pressure for the blade $i$ along the $j$ body axis.

The effect of the parameter that describes the position of the blade plane relative to the center of mass, the dimensionless ratio $k_{31}=r_{13} / r_{11}$, was analyzed together with stability indices that describe the device behavior.

The aim of the approach developed is to analyze the effect of the parameter $k_{31}$ on flight stability. With the objective of comparing the case where $k_{31}$ is different from zero with the case where $k_{31}$ is zero, the same aerodynamic model used in previous work [16] was adopted. The fact of considering the parameter $k_{31}$ implies the introduction of a great complexity because the number of terms of system of equations became extremely large.

The advantage of an analytical model like the one developed here is that stability limits can be found using an approximated approach, useful for the determination of the type of evolution and the influence of the main parameters.

\section{A. Mathematical Model}

The system analyzed is a pararotor flying in the autorotation regime. It is modeled as a cylindrical body with two identical blades that rotates at angular velocity $\omega$ and falls vertically at uniform speed $U_{\infty}$.

In pararotors with small-aspect-ratio wings, when the falling speed is very large, the trajectory is vertical, and it is not affected by the small changes in pararotor orientation, as was observed in steady flight of a pararotor with small-aspect-ratio wings in a vertical tunel studied previously [21]. Therefore, the rigid-body rotational dynamics can be decoupled from the center of mass dynamics.

The induced velocity $v_{i}$ was not directly included in the model, following similar assumptions of former studies [16]. Other studies [22] indicated that a real low-aspect-ratio rotary wing shows an induced power correction factor $\kappa$ of about 2 or greater. This fact

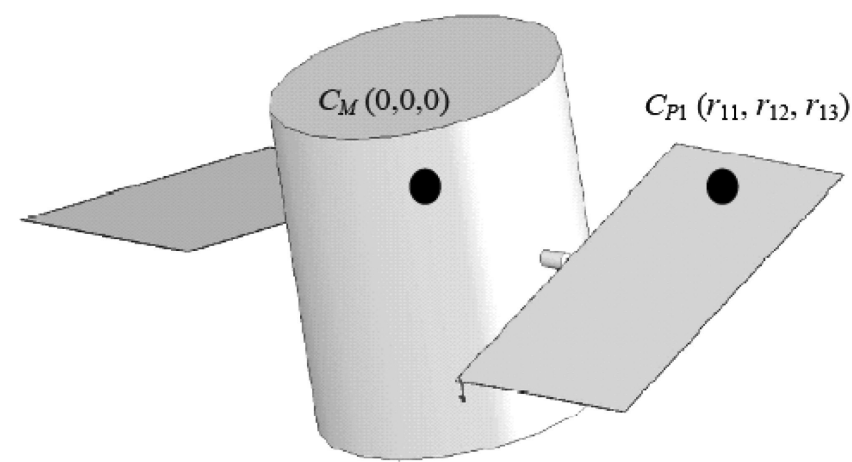

Fig. 1 System geometry scheme: center of mass $C_{M}$ position, and center of pressure $C_{P 1}$ of blade 1 . 
indicates that the relationships of descent rate and axial hovering velocity $v_{h}$ is greater than 2 , indicating a windmill brake state axial descend [23]. The current model focuses on the assumption of small induced velocity in the windmill brake state. Large induced power correction factors associated with low-aspect-ratio wings are consistent with this hypothesis. Vertical tunnel tests performed [21] using different small-aspect-ratio wings pararotors showed induced velocities of $15 \%$ of the descent velocity on average, evidence that the simplifying hypothesis is associated with a representative flight range.

The flow model for axial descent in a windmill brake state [23] indicates that the sensibility of induced velocity $v_{i}$ to descent rate decreases with $U_{\infty} / v_{h}$. This means that vertical velocity disturbances influence on induced velocity depends on the $v_{i} / v_{h}$ magnitude and thus on $\kappa$.

However, the effect of induced velocity is included in the values of lift, lift slope, and drag coefficients, $C_{L}, C_{L \alpha}$, and $C_{D}$, which are determined from experiments [15]. This experimental work was performed in operational conditions of a pararotor model, and so the effect of $v_{i}$ will result on an increment of the magnitudes of lift and drag if the freestream velocity over the blades is considered. As a consequence, the model will be developed associated with these experimental data that will define an operating range of validity, over which the lift slope can be approximated as linear. This range is defined in the incompressible regime. The model geometry is defined in Fig. 2. The body-fixed reference system [1-3] has its origin at the center of mass $C_{M}$ and directions $\boldsymbol{e}_{1}, \boldsymbol{e}_{2}, \boldsymbol{e}_{3}$. The axes [1-3] are the principal axes of the body, right-hand defined. The inertial reference system is $[X, Y, Z]$, right-hand defined; its axes have the directions $i$, $\boldsymbol{j}, \boldsymbol{k}$. The blades are located on an axis parallel to axis 1 that intersects axis 3 in the point named "B". Each blade $P_{1}$ and $P_{2}$ has a pitch angle $\beta_{1}$ and $\beta_{2}$, respectively.

The normal vectors associated to each blade are $\boldsymbol{n}_{1}=$ $\left[0, \sin \beta_{1}, \cos \beta_{1}\right], \boldsymbol{n}_{2}=\left[0,-\sin \beta_{2}, \cos \beta_{2}\right]$.

The rotation vector $\boldsymbol{\omega}=\left[\omega_{1}, \omega_{2}, \omega_{3}\right]^{T}$ is expressed in the body reference system. The gravity vector points $-\boldsymbol{k}$.

Moments defined in body axes are $\boldsymbol{M}=\left[M_{1}, M_{2}, M_{3}\right]^{T}$.

Euler equations in axes $\boldsymbol{e}_{i}$, with moments taken respect to the center of mass, are

$$
\begin{gathered}
\dot{\omega}_{1}-\frac{I_{2}-I_{3}}{I_{1}} \omega_{2} \omega_{3}=\frac{1}{I_{1}} M_{1} \\
\dot{\omega}_{2}+\frac{I_{1}-I_{3}}{I_{3}} \omega_{1} \omega_{3}=\frac{1}{I_{2}} M_{2} \\
\dot{\omega}_{3}-\frac{I_{1}-I_{2}}{I_{3}} \omega_{1} \omega_{2}=\frac{1}{I_{3}} M_{3}
\end{gathered}
$$

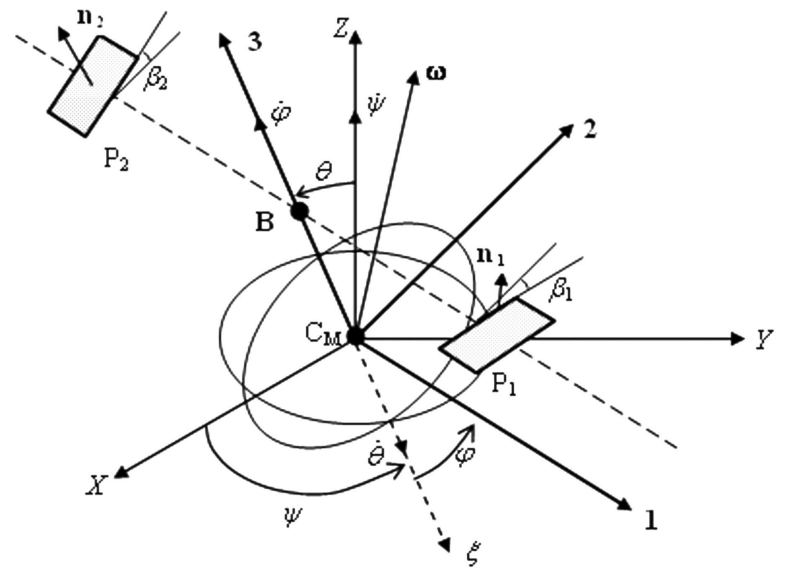

Fig. 2 System geometry: inertial reference system $(X, Y, Z)$, body-fixed reference system $(1,2,3)$, precession angle $\psi$, nutation angle $\theta$, spin angle $\varphi$, and angular velocity $\omega$.
The descent rate is expressed in axes $\boldsymbol{e}_{i}$ as $\boldsymbol{U}_{\infty}=$ $U_{\infty}[\sin \theta \sin \varphi, \sin \theta \cos \varphi, \cos \theta]^{T}$. We consider that the pararotor is flying near an equilibrium point defined by small nutation angles, which was observed in previous experimental work [21]. This equilibrium flight state can be described as a vertical descent with axis $\boldsymbol{e}_{3}$ mostly aligned with the gravity vector. The expression of the descent rate can be approximated by $\boldsymbol{U}_{\infty} \approx U_{\infty}[\theta \sin \varphi, \cos \varphi, 1]^{T}$.

The Newton equations that would complete the six-degree-offreedom rigid-body system dynamic is decoupled from Eq. (1), considering the case of a flight near an equilibrium point that is characterized by the small cross influence of translations and rotations, observed in vertical tunnel tests. As a consequence, this model is limited by this simplifying hypothesis.

The distance from the center of presure of blade $i(i=1,2)$ to the center of mass in axes $\boldsymbol{e}_{i}$, is $\boldsymbol{R}_{i}=\left[r_{i 1}, r_{i 2}, r_{i 3}\right]^{T}$. For simplicity, both blades are considered geometrically identical, which means that $r_{11}=-r_{21}, r_{12}=-r_{22}$, and $r_{13}=r_{23}$.

The relative velocity of the fluid to blade $i(i=1,2)$, considering a vertical descent rate of the body, $\boldsymbol{U}_{\infty}=U_{\infty} \boldsymbol{k}$ is defined as

$$
\boldsymbol{V}_{r i}=-\boldsymbol{\omega} \times \boldsymbol{R}_{i}+\boldsymbol{U}_{\infty}
$$

The lift and drag forces can be expresed, for each blade, as

$$
\begin{gathered}
\boldsymbol{L}_{i}=\frac{1}{2} \rho S C_{L \alpha} \alpha_{i}\left|\boldsymbol{V}_{r i}\right|^{2} \boldsymbol{e}_{L i} \\
\boldsymbol{D}_{i}=\frac{1}{2} \rho S C_{D}\left|\boldsymbol{V}_{r i}\right|^{2} \boldsymbol{e}_{D i}
\end{gathered}
$$

where $\boldsymbol{e}_{L i}$ is the unit vector aligned to lift force, $\boldsymbol{e}_{D i}$ is the unit vector aligned to drag force, $\alpha_{i}$ is the angle of attack of each blade, $S$ is the blade area, $\rho$ is the air density, $C_{L \alpha}$ is the slope of the blade curve lift (with respect to $\alpha$ ), and $C_{D}$ is the blade drag coefficient.

The unit vector $\boldsymbol{e}_{L i}$, can be defined, for each blade, as

$$
\boldsymbol{e}_{L i}=(-1)^{i} \frac{\boldsymbol{e}_{1} \times V_{r i}}{\left|\boldsymbol{e}_{1} \times V_{r i}\right|}
$$

This expression can be approximated, considering that $\boldsymbol{V}_{r i}$ is perpendicular to $\boldsymbol{e}_{1}$, which is consistent with Eq. (2) and the flight conditions defined as a hypothesis:

$$
\boldsymbol{e}_{L 1} \cong(-1)^{i} \frac{\boldsymbol{e}_{1} \times \boldsymbol{V}_{r 1}}{\left|\boldsymbol{V}_{r 1}\right|}
$$

The unit vector $\boldsymbol{e}_{D i}$, can be defined, for each blade, as

$$
\boldsymbol{e}_{D i}=\frac{\boldsymbol{V}_{r i}}{\left|\boldsymbol{V}_{r i}\right|}
$$

The angle of attack for each blade can be defined as

$$
\sin \alpha_{i}=\frac{\boldsymbol{n}_{i} \cdot \boldsymbol{V}_{r i}}{\left|\boldsymbol{V}_{r i}\right|}
$$

If we consider the previous expressions, we can determine the aerodynamic forces acting on each blade. These expressions can be approximated taking into account the following assumptions and neglecting second- and higher-order terms (when a term contains a multiplication of more than two small factors, it is neglected).

1) Both wings have identical pitch angle.

2) The angles of attack and the angles of incidence are all small, say less than $0.25 \mathrm{rad}$.

3) The component along axis 1 of the relative velocity to the blade has a neglectable aerodynamic influence.

4) The distance from the aerodynamic center of the blades to the center of mass in the direction perpendicular to the blade span is small. 
5) The angular velocity along axes 1 and $2, \omega_{1}$ and $\omega_{2}$, are small compared with $\omega_{3}$.

For simplicity, the dimensionless vertical velocity to blade velocity, $k=U_{\infty} /\left(\omega_{3} r_{11}\right)$, is introduced. Also, a change of variables is defined by $x_{1}=\omega_{1} / \omega_{3}$ and $x_{2}=\omega_{2} / \omega_{3}$. The aerodynamic forces that act over the blades $P_{1}$ and $P_{2}$ can be written [9] expressed in reference frame [1-3] as

$$
\begin{gathered}
\boldsymbol{L}_{1} \cong \frac{1}{2} \rho S C_{L \alpha} r_{11}^{2} \omega_{3}^{2}\left(-\beta_{1}+x_{2}+k\right)\left(\begin{array}{c}
0 \\
\left(x_{2}+k\right) \\
1
\end{array}\right) \\
\boldsymbol{L}_{2} \cong \frac{1}{2} \rho S C_{L \alpha} r_{11}^{2} \omega_{3}^{2}\left(-\beta_{2}+x_{2}+k\right)\left(\begin{array}{c}
0 \\
\left(x_{2}-k\right) \\
1
\end{array}\right) \\
\boldsymbol{D}_{1} \cong \frac{1}{2} \rho S C_{D} r_{11}^{2} \omega_{3}^{2}\left(\begin{array}{c}
x_{2} k_{31}-1 \\
x_{2}-k_{21}+k
\end{array}\right) \\
\boldsymbol{D}_{2} \cong \frac{1}{2} \rho S C_{D} r_{11}^{2} \omega_{3}^{2}\left(\begin{array}{c}
x_{2} k_{31}+1 \\
0 \\
x_{2}+k_{21}+k
\end{array}\right)
\end{gathered}
$$

The aerodynamic moments acting on the body itself were neglected, considering that the nutation angles are small, and that the center of pressure of the body is close to the center of mass of the pararotor. The moment of the aerodynamic forces of the blades, taking into account Eqs. (8) and (9) is

$$
\boldsymbol{M}=\sum_{i=1}^{i=2}\left[\boldsymbol{R}_{i} \times\left(\boldsymbol{L}_{i}+\boldsymbol{D}_{i}\right)\right]
$$

The moments along the $j$ axis can be expressed as

$$
M_{j}=\frac{1}{2} \rho S r_{11}^{3} \omega_{3}^{2} C_{M j}
$$

where the moment coefficients named $C_{M i}$ derived from Eq. (10) are

$$
\begin{array}{r}
C_{M 1}=C_{L \alpha} k_{31}\left[\left(\beta_{1}+\beta_{2}\right) x_{2}+\left(\beta_{1}-\beta_{2}\right) k-4 x_{2} k\right] \\
+C_{L \alpha} k_{21}\left[-\beta_{1}+\beta_{2}+2 x_{2}\right]+C_{D} k_{31}\left[-4 x_{1} k_{31}\right] \\
C_{M 2}=-C_{L \alpha}\left[-\beta_{1}+\beta_{2}+2 x_{2}\right]-2 C_{D} x_{2} \\
C_{M 3}=C_{L \alpha}\left[-\left(\beta_{1}-\beta_{2}\right) x_{2}-\left(\beta_{1}+\beta_{2}\right) k+2\left(x_{2}\right)^{2}+2 k^{2}\right] \\
+2 C_{D}\left[x_{1} k_{31}\right]
\end{array}
$$

For $r_{13}=0$ (the blades plane is aligned with the center of mass), and neglecting the higher-order terms, the equations developed in $[10,15]$ are recovered.

\section{B. Linearization}

To study the dynamic behavior of the pararotor around an equilibrium point, the system given by Eqs. (1) and (11) is linearized.

As analyzed in $[15,16]$, it is considered that $\omega_{3}$ remains constant when the system is perturbed around an equilibrium point. In a smallaspect-ratio blade pararotor, the induced power correction factor $\kappa$ is considerably larger than in large-aspect-ratio rotary-wing decelerators. This fact derives in a windmill brake state, where $U_{\infty}$ is large compared with $v_{h}$, and $\omega_{3}$ is of a large magnitude, which is confirmed by experimental observation. As a first approximation, the sensitivity of $\omega_{3}$ to $U_{\infty}$ variations can be taken as a second-order effect. As a consequence of these considerations, $\omega_{3}$ and $U_{\infty}$ are taken constant in the proximity of the equilibrium flight. Then, Eq. (1c) can be uncoupled from the rest of Euler's equations, as an approximation to the problem.

The column vector of state variables is defined as $x(t)=$ $\left[x_{1}(t), x_{2}(t)\right]^{T}$, taking into account the decoupling simplifications. The simplified equations of the system dynamics are a nonlinear system that can be written in dimensionless variables as

$$
\frac{\mathrm{d} \boldsymbol{x}}{\mathrm{d} T}=\boldsymbol{F}(\boldsymbol{x}, T)=\left[\frac{M_{1}}{I_{1} \omega_{3}^{2}}+\left(\frac{I_{2}-I_{3}}{I_{1}}\right) x_{2}, \frac{M_{2}}{I_{2} \omega_{3}^{2}}-\left(\frac{I_{1}-I_{3}}{I_{2}}\right) x_{1}\right]^{T}
$$

The initial conditions of the problem are defined as: $x(0)=$ $\boldsymbol{x}_{0}=[0,0]^{T}$.

The trim problem is defined as $\boldsymbol{F}\left(\boldsymbol{x}_{e}\right)=0$, where $\boldsymbol{x}_{e}=\left[x_{e 1}, x_{e 2}\right]^{T}$ is the equilibrium value of the state vector.

The essence of linearization is the assumption that the motion can be considered as a perturbation about a trim or equilibrium condition; provided that the perturbations are small, the function $\boldsymbol{F}$ can usually be expanded in terms of the motion and control variables and the response written in the form $\boldsymbol{x}=\boldsymbol{x}_{e}+\Delta \boldsymbol{x}$, where $\Delta \boldsymbol{x}$ is the disturbed estate vector.

The system of equations [Eq. (15)] can be linearized by expansion in the neighborhood of the equilibrium point as follows:

$$
\frac{\mathrm{d} \boldsymbol{x}}{\mathrm{d} t}=\frac{\mathrm{d}\left(\boldsymbol{x}_{e}+\Delta \boldsymbol{x}\right)}{\mathrm{d} t}=\boldsymbol{F}\left(\boldsymbol{x}_{e}\right)+\left(\frac{\partial F_{i}}{\partial x_{j}}\right)_{\boldsymbol{x}_{e}}^{i, j=1,2} \Delta \boldsymbol{x}+O\left(|\Delta \boldsymbol{x}|^{2}\right)
$$

where the linear problem is defined as

$$
\frac{\mathrm{d} \Delta \boldsymbol{x}}{\mathrm{d} t}=\left(\frac{\partial F_{i}}{\partial x_{j}}\right)_{\boldsymbol{x}_{\varepsilon}}^{i, j=1,2} \Delta \boldsymbol{x}=[\boldsymbol{A}] \Delta \boldsymbol{x}
$$

where $\boldsymbol{A}$ is the characteristic matrix of the system of equations.

The constant matrix consequence of the linealization can be expressed as

$$
\begin{aligned}
\boldsymbol{A} & =\left[\begin{array}{ll}
A_{11} & A_{12} \\
A_{21} & A_{22}
\end{array}\right] \\
& =\left[\begin{array}{cc}
\frac{1 \rho S r_{11}^{3}}{2} I_{1}\left(-4 C_{D} k_{31}^{2}\right) & \frac{I_{2}-I_{3}}{I_{1}}+\frac{1 \rho S r_{11}^{3}}{2} \\
I_{1} & {\left[C_{L \alpha} k_{31}\left(\beta_{1}+\beta_{2}-4 k\right)+2 C_{L \alpha} k_{21}\right]} \\
-\frac{I_{1}-I_{3}}{I_{2}} & \frac{\rho S r_{11}^{3}}{I_{2}}\left(-C_{L \alpha}-C_{D}\right)
\end{array}\right]
\end{aligned}
$$

For $k_{31}=0$, matrix $A$ is coincident with the one developed in $[15,16]$.

\section{Equilibrium Points}

Neglecting the higher-order terms, the equilibrium points are attained are

$$
\begin{gathered}
x_{e 2}=\frac{2 K_{1} C_{L \alpha} \delta_{\beta}\left(-k_{31} k+k_{21}\right)}{2 K_{1} C_{L \alpha}\left(k_{31} \beta_{0}-2 k_{31} k+k_{21}\right)+\frac{I_{2}-I_{3}}{I_{1}}} \\
x_{e 1}=\frac{2 K_{2}\left[C_{L \alpha} \delta_{\beta}-\left(C_{D}+C_{L \alpha}\right) x_{e 2}\right]}{\frac{I_{1}-I_{3}}{I_{2}}}
\end{gathered}
$$

where

$$
K_{i}=\frac{1}{2} \frac{\rho S r_{11}^{3}}{I_{i}}
$$

with $i=1,2$.

If we consider the terms $K_{i} C_{L \alpha}$, that appear in Eqs. (18) and (19), it can be observed that it is similar to the Lock number [24]. As a consequence, these terms can be interpreted as a nondimensional 
Table 1 Signs of equilibrium points in terms of principal moments of inertia and $k_{31}$

\begin{tabular}{lccc}
\hline \hline & & \multicolumn{2}{c}{ Equilibrium points } \\
\cline { 3 - 4 } Moment of inertia & $k_{31}$ & $x_{e 1}$ & $x_{e 2}$ \\
\hline$I_{3}>I_{2}, I_{1}$ & $>0$ & $<0$ & $>0$ if $k_{31} k>k_{21}$ \\
$I_{3}>I_{2}, I_{1}$ & $=0$ & $<0$ & $<0$ \\
$I_{3}>I_{2}, I_{1}$ & $<0$ & $<0$ & $<0$ \\
$I_{3}<I_{2}, I_{1}$ & $>0$ & $>0$ & $<0$ if $k_{31} k<k_{21}$ \\
$I_{3}<I_{2}, I_{1}$ & $=0$ & $>0$ & $>0$ \\
$I_{3}<I_{2}, I_{1}$ & $>0$ & $>0$ & $>0$ \\
$I_{2}<I_{3}<I_{1}$ & $=0$ & $<0$ & $>0$ \\
$I_{2}<I_{3}<I_{1}$ & $>0$ or $<0$ & $>0$ or $<0$ & $>0$ or $<0$ \\
$I_{1}<I_{3}<I_{2}$ & $=0$ & $>0$ & $<0$ \\
$I_{1}<I_{3}<I_{2}$ & $>0$ or $<0$ & $>0$ or $<0$ & $>0$ or $<0$ \\
\hline \hline
\end{tabular}

scaling coefficient, giving the ratio of aerodynamic to inertia forces acting on a rotor blade, associated with the response sensitivity of the system. Table 1 shows the effects of $k_{31}$ for different moments of inertia ratios on the sign of equilibrium points $\left(x_{e 1}, x_{e 2}\right)$.

\section{Stability Analysis}

The eigenvalues of matrix $\boldsymbol{A}, \lambda_{1}$ and $\lambda_{2}$, dictate the system asymptotic stability under the assumed hypothesis. These eigenvalues are given by

$$
\begin{aligned}
\lambda_{1,2} & =\frac{1}{2}\left(T_{r} \pm \sqrt{\Delta}\right) \\
& =\frac{\left(A_{11}+A_{22}\right) \pm \sqrt{\left(A_{11}+A_{22}\right)^{2}+4\left(A_{12} A_{21}-A_{11} A_{22}\right)}}{2}
\end{aligned}
$$

where $\Delta=T_{r}^{2}-4 D_{e t}$, and $\operatorname{Tr}$ and $D_{e t}$ are the trace and the determinant of matrix $\boldsymbol{A}$, respectively.

A linear system is asymptotically stable if and only if the real part of the eigenvalues of matrix $\boldsymbol{A}$ is negative [25]. The eigenvalues also define the evolution of the trajectories of the system: either stable or unstable. The eigenvectors define the type of evolution (node, spiral, focus). In the case of a two-variable systems, the following stable cases appear $[10,15]$.

1) A stable node $\lambda_{1}<0$ and $\lambda_{2}<0 ; \lambda_{1,2}$ are real and different, $T_{r}<0, \Delta>0$.

2) A stable spiral $\lambda_{12}=p \pm q i, p<0 ; q \neq 0, i$ is the unit imaginary number, $T_{r}<0, \Delta<0$.

3) A stable focus $\lambda_{1}=\lambda_{2}<0 ; T_{r}<0, \Delta=0$.

The stability limits are defined by the conditions $T_{r}=0$ and $\Delta=0$

The eigenvectors associated with eigenvalues from Eq. (20), $v_{1,2}$, define the system trajectories in a phase-plane analysis of the secondorder system.

\section{Stable Node}

To attain this condition, the Routh-Hurwitz criteria must be satisfied:

$$
\begin{gathered}
T_{r}=2 K_{2}\left(C_{L \alpha}+C_{D}\right)+2 K_{1} C_{D} k_{31}^{2}>0 \\
-D_{e t}=\frac{\left(I_{3}-I_{2}\right)\left(I_{3}-I_{1}\right)}{I_{2} I_{1}}-K_{1}\left(C_{L \alpha} k_{31}\left(\beta_{1}+\beta_{2}-4 k\right)\right. \\
\left.+2 C_{L \alpha} k_{21}\right)\left(\frac{I_{3}-I_{1}}{I_{2}}\right)-c>0
\end{gathered}
$$

where $c=2 K_{1} K_{2} C_{D} k_{31}^{2}\left(C_{L \alpha}+C_{D}\right)$, which is neglected because it is a higher-order term in the Eq. (22).

It can be seen that the sole condition of $k_{31}$ being different from 0 contributes to increase the stability as given by Eq. (21). In the stability limit $T_{r}=0$, the trajectory is a center, and that occurs when $k_{31}=0$, and $C_{L \alpha}=0$ and $C_{D}=0$. This situation coincides with the classical problem of a rotating body in a vacuum.

If the expression given by Eq. (22) is rearranged, it can be expressed as

$$
\left(I_{3}-I_{2}\right)\left(I_{3}-I_{1}\right)\left(1-N_{e}^{\prime}\right)>0
$$

where the stability number is defined as

$$
N_{e}^{\prime}=I_{a} C_{L \alpha} \frac{k_{31}\left(\beta_{0}-2 k\right)+k_{21}}{I_{3}-I_{2}}
$$

and

$$
I_{a}=\rho S r_{11}^{3}
$$

From Eq. (21), two conditions can be defined: if $I_{2}<I_{3}, I_{1}$ or $I_{3}<I_{2}$, $I_{1}$, then $N_{e}^{\prime}$ must be less than 0 , and if $I_{2}<I_{3}<I_{1}$ or $I_{1}<I_{3}<I_{2}$, then $N_{e}^{\prime}$ must be greater than 0 . Considering that $\alpha>\beta_{0}$ by geometric definition, the effect over the condition Eq. (22) is as follows.

1) When $I_{2}<I_{3}$, values of $k_{31}$ greater than 0 contribute to make $D_{e t}$ greater than 0 .

2) When $I_{3}<I_{2}$, then values of $k_{31}$ less than 0 contributes to make $D_{e t}$ greater than 0 .

From Eq. (23), it can be seen that, when $I_{2}<I_{3}<I_{1}$, the value of $N_{e}^{\prime}$ must be greater than 1 to fulfill the condition, and then values of $k_{31}$ greater than 0 contribute to the criteria.

For this case, the phase portrait would show trajectories moving directly toward and converge to the equilibrium solution (node). The eigenvectors indicate trajectories directions; when near the equilibrium point will be approximately in the same direction as the eigenvector associated with the eigenvalue with the smaller absolute value, farther away the equilibrium point, they would bend toward the direction of the eigenvector of the eigenvalue with the larger absolute value.

\section{Stable Spiral}

This case occurs when $\Delta<0$. This condition can be written as

$$
\left(A_{11}+A_{22}\right)^{2}-4\left(A_{11} A_{22}-A_{12} A_{21}\right)<0
$$

When spiral trajectory is given, the stability of the system is guaranteed if $T_{r}>0$, and so the sole condition that $k_{31}$ be different from 0 fulfills that condition, as far as $C_{L \alpha}, C_{D}$, are generally positive.

If $k_{31}$ is equal to zero, then Eq. (26) can be written as [15]

$$
\frac{\left(I_{3}-I_{2}\right)^{2}}{k_{21}^{2} I_{2}^{2}}\left[N_{e}^{\prime 2}-k_{e}\left(1-N_{e}^{\prime}\right)\right]<0
$$

where the dimensionless parameter $k_{e}$ is defined as

$$
k_{e}=4 k_{21}^{2} \frac{I_{2}}{I_{1}} \frac{I_{3}-I_{1}}{I_{3}-I_{2}}
$$

For this case, the phase portrait would show spirals trajectories moving toward and converge to the equilibrium solution (spiral point).

\section{Stable Focus}

This type of trajectory will arise when the condition is defined as

$$
\Delta=T_{r}^{2}-4 D_{e t}=0
$$

that is

$$
\left(A_{11}+A_{22}\right)^{2}-4\left(A_{11} A_{22}-A_{12} A_{21}\right)=0
$$


When stable focus is given, the stability of the system is guaranteed if $T_{r}>0$, and so the sole condition that $k_{31}$ be different from 0 fulfills that condition.

The eigenvalues become

$$
\lambda_{1}=\lambda_{2}=A_{11}+A_{22}
$$

For this case, the phase portrait would show logaritmic spiral trajectories moving toward and converge to the equilibrium solution (focus point).

\section{E. Stability Regions}

Because the signs of the differences between moments of inertia have an influence in the stability limits, their relative values should be taken into account. The parameters $N_{e}^{\prime}, k_{e}$ can account for the effects of principal moments of inertia ratios and are able to define stability regions of the system analyzed. These parameters represent a more general case of the parameters presented in $[15,16]$.

The six possible configurations can be reduced to four cases, as discussed in the following paragraphs.

\section{Case 1: Spinning Motion Close to the Major Axis of Inertia, Inertial Stability}

Two configurations are included in this case, $I_{1}<I_{2}<I_{3}$ and $I_{2}<I_{1}<I_{3}$; thus, according to Eq. (23), the stability region is defined by the condition $N_{e}^{\prime}<1$.

Because both $I_{2}<I_{3}$ and $I_{1}<I_{3}, k_{e}$ are bigger than 0 , and because $N_{e}^{\prime}$ can take either positive or negative values in a general case, only the first and second quadrants of the plane $\left(N_{e}^{\prime}, k_{e}\right)$ are allowed (Fig. 3.

The limit for the region of spirals (focuses) is given by $\Delta=0$. If $k_{31}=0$, then $\Delta$ is given by $N_{e}^{\prime 2}-k_{e}\left(1-N_{e}^{\prime}\right)=0$ [15]. Then, $k_{e}=N_{e}^{\prime 2} /\left(1-N_{e}^{\prime}\right)$, and the region of spirals is attained when $k_{e}>N_{e}^{\prime 2} /\left(1-N_{e}^{\prime}\right)$.

When $N_{e}^{\prime}>1$, with large aerodynamic effects compared to $I_{3}-I_{2}$ [see Eq. (24)], the system is unstable. This situation does not happen when there are no aerodynamic forces over the body, that is, in the classical problem of a solid body rotating in a vacuum, whose motion is always stable under the condition $I_{1}, I_{2}<I_{3}$.

\section{Case 2: Spinning Motion Close to the Axis of Lower Inertia, Inertial} Stability

Two configurations are included in this case, $I_{3}<I_{1}<I_{2}$ and $I_{3}<I_{2}<I_{1}$, and therefore $k_{e}>0$. Thus, the first and second quadrants of the plane $\left(N_{e}^{\prime}, k_{e}\right)$ are allowed. This situation is shown in Fig. 4.

When $N_{e}^{\prime}=0$, the system follows a center-type evolution. This situation corresponds to the case where $C_{L \alpha}=0$ and is stable, as expected [10].

It can be seen that, when $N_{e}^{\prime}<1$, the system is stable.

From Eq. (27), it can be seen that $N_{e}^{\prime}$ increases when $k_{31}$ increases. Therefore, while $k_{31}$ increases, the system approaches the stability limit.

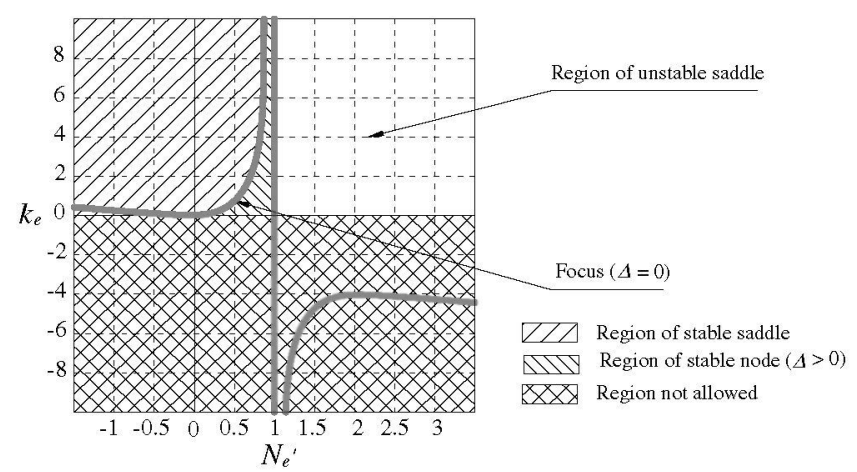

Fig. 3 Types of trajectories for the case $I_{1}, I_{2}<I_{3}$ in the $N_{e}^{\prime}, k_{e}$ plane. The first and second quadrants are allowed.
3. Case 3: Spinning Motion Close to the Axis of Intermediate Inertia $\left(I_{2}<I_{3}<I_{1}\right)$, Aerodynamic Stabilization

In this case $\left(I_{2}<I_{3}<I_{1}\right)$, according to Eq. (24), the condition $N_{e}^{\prime}>1$ must hold for a stable system. Furthermore, by definition, $k_{e}<0$; therefore, only the third and fourth quadrants of the plane $\left(N_{e}^{\prime}, k_{e}\right)$ are allowed.

Considering Eq. (23), it is possible to reach the stable region if the following condition is given:

$$
k_{21}>\frac{I_{3}-I_{2}}{I_{a} C_{L \alpha}}-k_{31}\left(\beta_{0}-2 k\right)
$$

This means that the value of $k_{31}$ is determinant for the stability of the system. Therefore, under specific conditions, the system can be stable when it is spinning around an axis close to the intermediate inertia axis. This is due to the stabilization effect of the gravity applied at the center of mass, as it can be seen by the effect introduced by $k_{31}$ on Eq. (31).

\section{Case 4: Spinning Motion Close to the Axis of Intermediate Inertia $\left(I_{1}<I_{3}<I_{2}\right)$, Gravity Stabilization}

In this case $\left(I_{1}<I_{3}<I_{2}\right)$, according to Eq. (23), the condition $N_{e}^{\prime}>1$ must be fulfilled for stable motion. Furthermore, by definition, $k_{e}$ is smaller than 0 ; therefore, only the third and fourth quadrants of the plane $\left(N_{e}^{\prime}, k_{e}\right)$ are allowed (see Fig. 5).

In this case, if $k_{31}=0$, it is not possible to attain a stable configuration, as was established previously $[15,16]$.

Considering Eq. (22), it is possible to reach the stable region if the following condition is given:

$$
k_{21}>\frac{I_{3}-I_{2}}{I_{a} C_{L \alpha}}-k_{31}\left(\beta_{0}-2 k\right)
$$

This means that a value of $k_{31}$ big enough to fulfill Eq. (32) gives a stable device. This is due to the stabilizing effect of the aerodynamic forces generated by the blades that can be reinforced by the stabilization effect of the gravity applied at the center of mass, as can be seen by the effect introduced by $k_{31}$ on Eq. (32). Without that contribution of the aerodynamic forces, the system would be unstable, as is the case for rotation in vacuum.

In terms of practical configurations, pararotors that meet the condition given by Eq. (32) are useless or impractical $\left(k_{31}=100\right)$. In general, practical configurations (that may be $k_{31}=10$ or smaller) are unstable when $I_{1}<I_{3}<I_{2}$.

\section{F. Numerical Examples}

Numerical results of the stability analysis are presented in this section, according to the cases presented in Sec. II.E. To develop them, an aluminium solid body composed of both a cylinder and blades with different relative dimensions that produce different relations between the principal moments of inertia is considered. The main common model parameters used for the example are summarized in Table 2.

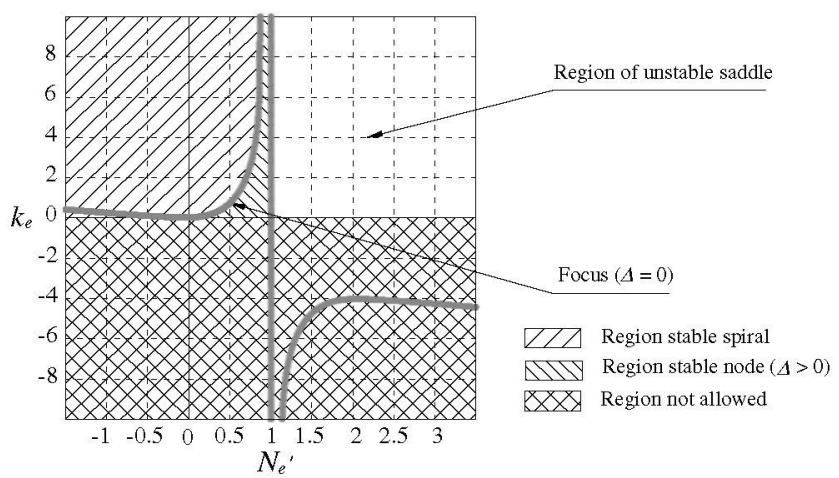

Fig. 4 Types of trajectories for the case $I_{3}<I_{2}, I_{3}$ in the $N_{e}^{\prime}, k_{e}$ plane. The first and second quadrants are allowed. 
Table 2 Common parameters of the numerical examples

\begin{tabular}{lc}
\hline \hline Parameter & Value \\
\hline$C_{L \alpha}$, & $3.4 \mathrm{rad}^{-1}$ \\
$C_{D}$ & 0.07 \\
$\rho$ & $1.21 \mathrm{~kg} / \mathrm{m}^{3}$ \\
$\omega_{0}$ & $291.4 \mathrm{rad} / \mathrm{s}$ \\
$S$ & $0.012 \mathrm{~m}^{2}$ \\
$r_{11}, r_{21}$ & $0.1 \mathrm{~m}$ \\
$r_{12}, r_{22}$ & $0.034 \mathrm{~m}$ \\
$\beta_{1}$ & $0.1 \mathrm{rad}$ \\
$\beta_{2}$ & $0.17 \mathrm{rad}$ \\
$\delta_{\beta}$ & $0.035 \mathrm{rad}$ \\
$U_{\infty}$ & $14.1 \mathrm{~m} / \mathrm{s}$ \\
\hline \hline
\end{tabular}

In the examples, the four cases presented in the stability analysis were considered to qualitatively describe the stability analysis. In Table 2, the common parameters of numerical examples studied are presented, taking as a reference measurements done in the context of experimental test.

In Table 3, the principal moments of inertia of the cases taken for this analysis are shown. These examples are representative cases of those presented previously, chosen by their moments of inertia relationship. Case 1.b presents an example of the $I_{1}<I_{2}<I_{3}$ case where stability is weak due to the proximity of $I_{2}$ and $I_{3}$, in contrast with case 1 .a, where stability is stronger. Cases 3 and 4 were found to be in general unstable, with values of $k_{31}$ in the range of -1 to 1 .

In Table 4, the real part of the largest eigenvalue of the characteristic matrix of the linearized system is presented to show the effect of $k_{31}$ on the stability. In Fig. 6, these cases are illustrated. In Table 4 , the effect of $k_{31}$ on the value of $\left(N_{e}^{\prime}, k_{e}\right)$ is presented.

\section{Experiments}

In this section, some experiments that have been carried out to validate the analytical model are presented.

\section{A. Experimental Setup}

To test the pararotor behavior, an horizontal closed-circuit wind tunnel with rectangular closed test section ( $1.4 \mathrm{~m}$ wide to $1.0 \mathrm{~m}$ high) has been used.

A schematic description of the tested models and the general wind tunnel setup is shown in Fig. 7. The pararotor was placed in the test section, fixed to a load cell. A bearing allowed a three-degree-offreedom rotation (of limited magnitude) of the model and prevented translation. This bearing was placed in such a way that the center of mass is kept in the plane of symmetry of the bearing and in its center. The axis perpendicular to blade plane of the pararotor was aligned with the wind flow to simulate the device descent. A load cell was rigidly fixed to the wind tunnel to measure the model drag.

The tested models consisted of a cylindrical main body with two rectangular flat blades attached. The main body was a $90-\mathrm{mm}$-diam, 92-mm-high aluminum cylinder. The rectangular aluminum blades were $91 \mathrm{~mm}$ span, $141 \mathrm{~mm}$ chord, and $1 \mathrm{~mm}$ thick. The position of the plane containing the blades (rotor plane) can be changed $200 \mathrm{~mm}$ downstream or upstream the center of mass. The blade pitch was set before each test. The parameter $r_{11}$ was estimated to be $0.09 \mathrm{~m}$.

Table 5 shows the moments of inertia and weights of each model configuration.
Table 4 Numerical examples of effect of $k_{31}$ on $\left(\lambda_{r e 1}\right)$

\begin{tabular}{cccccc}
\hline \hline$k_{31}$ & Case 1.a & Case 1.b & Case 2 & Case 3 & Case 4 \\
\hline 1.0 & -0.0130 & -0.0130 & -0.0100 & 0.5848 & 0.6663 \\
0.5 & -0.0118 & -0.0118 & -0.0097 & 0.5893 & 0.6530 \\
0.0 & -0.0114 & -0.0114 & -0.0096 & 0.5935 & 0.6385 \\
-0.5 & -0.0118 & 0.1336 & -0.0097 & 0.5976 & 0.6229 \\
-1.0 & -0.0130 & 0.1984 & -0.0100 & 0.6015 & 0.6061 \\
\hline \hline
\end{tabular}

\section{B. Meassurents and Instrumentation}

The parameters that were measured in the tests and the instruments used are summarized in Table 6.

\section{Experimental Results}

Experiments showed in all of the cases a steady-state flight with $e_{3}$ aligned with the wind flow direction. The angular deviation of $e_{3}$ with respect to flow direction was of a negligible magnitude in any case.

The measured stream velocity $U_{\infty}$ and angular velocity $\omega_{3}$ are shown in Table 7. In this table, the parameters $N_{e}^{\prime}$ and $k_{e}$ were calculated in base to the experimental results using Eqs. (24) and (28), respectively.

\section{Results Analysis}

The experimental results showed that, in all cases, the equilibrium position was maintained, so that they were stable, with a steady-state rotation characterized by $e_{3}$ aligned to the wind flow direction. Thus, it could be seen that magnitudes of $k_{31}\left(k_{31}=0.2\right)$ in the test model did not affect stability in a significative way.

The parameters $N_{e}^{\prime}$ and $k_{e}$ for to the tested models correspond to stable behavior, as predicted by the analytical model; Table $7\left(N_{e}^{\prime}, k_{e}\right)$ values belong to the stable region defined in Fig. 2.

\section{E. Measurement Uncertainties}

The uncertainties of the measurement parameters were evaluated taking into account the reproducibility of tests performed in similar conditions and the uncertainties derived of the instruments used. The absolute values of the uncertainties that combines both types of contributions for $U_{\infty}$ and $\omega_{3}$ were $U_{U_{\infty}}=0.02 \mathrm{~m} \cdot \mathrm{s}^{-1} \mathrm{y}$ $U_{\omega 3}=0.01 \mathrm{rad} \cdot \mathrm{s}^{-1}$, respectively.

\section{Results}

The developed analytical model indicates, as expected, that pararotor stability is primarily defined by the relationship between the inertial moments of the body. This relationship can be classified in four cases given by $I_{1}, I_{2}<I_{3} ; I_{3}<I_{1}, I_{2} ; I_{1}<I_{3}<I_{2}$; and $I_{2}<I_{3}<I_{1}$. Depending on the inertia axis, close to which the body spins, different types (nodes, spirals, focuses) of trajectories appear at different regions of the stability diagram.

The model developed is in all terms congruent with the results of previous work $[15,16]$

The analytical model presented in this work depicts the main characteristics of the dynamic behavior of the pararotor near the equilibrium point. Every conclusion is given under the assumptions considered and the simplifications made.

When the distance between the center of mass and the plane that contains the blades is small (that is described by $k_{31}$ ), the stability is not affected by this parameter. When the distance between the center of mass and the plane that contains the blades is about the same as the distance from the axis of rotation to the center of the blades, or larger, the stability is affected.

Table 3 Principal moments of inertia of numerical cases adopted

\begin{tabular}{cccccc}
\hline \hline$k_{31}$ & Case 1.a & Case 1.a & Case 2 & Case 3 & Case 4 \\
\hline$I_{1}, \mathrm{~kg} \cdot \mathrm{m}^{2}$ & $6.31 \cdot 10^{-4}$ & $6.31 \cdot 10^{-4}$ & $22.1 \cdot 10^{-4}$ & $26.3 \cdot 10^{-4}$ & $6.31 \cdot 10^{-4}$ \\
$I_{2}, \mathrm{~kg} \cdot \mathrm{m}^{2}$ & $22.1 \cdot 10^{-4}$ & $22.1 \cdot 10^{-4}$ & $26.3 \cdot 10^{-4}$ & $6.31 \cdot 10^{-4}$ & $26.3 \cdot 10^{-4}$ \\
$I_{3}, \mathrm{~kg} \cdot \mathrm{m}^{2}$ & $26.3 \cdot 10^{-4}$ & $22.5 \cdot 10^{-3}$ & $6.31 \cdot 10^{-4}$ & $22.1 \cdot 10^{-4}$ & $22.1 \cdot 10^{-4}$ \\
\hline \hline
\end{tabular}




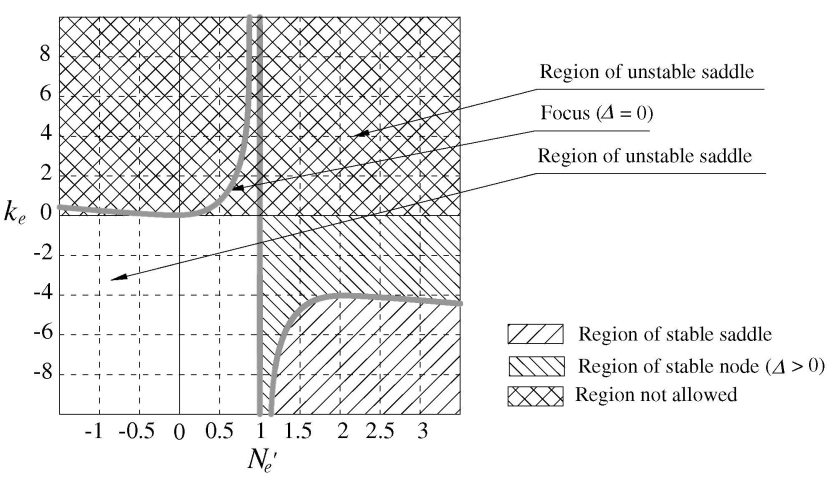

Fig. 5 Types of trajectories for $I_{1}<I_{3}<I_{2}$ or $I_{2}<I_{3}<I_{1}$ in the $N_{e}^{\prime}$, $k_{e}$ plane. The third and fourth quadrants are allowed.

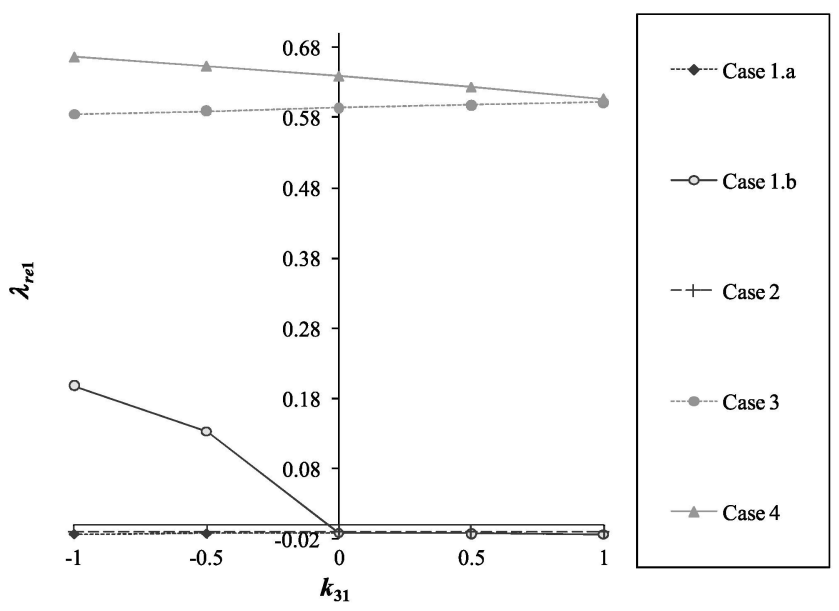

Fig. 6 Effect of $k_{31}$ on the value of the real part of the largest eigenvalue of the numerical examples for the linearized systems.

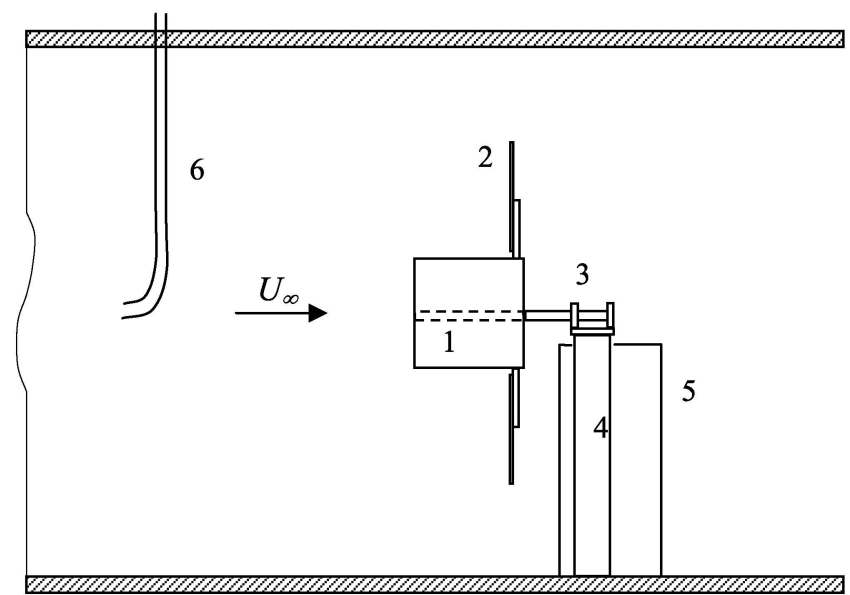

Fig. 7 Description of the test chamber setup. The numbers indicate test model body (1), test model blades (2), rotation axis (3), balance (4), balance fairing, (5), and pitot tube (6).

A number of numerical cases have been considered, varying the set of parameters of the configuration to drawn some conclusions. Thus, taking into account the relationship of inertial moments and analyzing the effect of $k_{31}$ on stability, the following can be seen, according to the analytical model developed.

1) When rotation takes place around or close to the principal axes or largest inertia (case 1 or 2) $I_{1}, I_{2}<I_{3}$ or $I_{3}<I_{1}, I_{2}$, positive values of $k_{31}$ (that means that the center of mass is under the plain of the blades) contribute to the system stability, reinforcing the tendency to asymptotic stability. The magnitude of this effect depends on distance from stability limits. When the configuration of the pararotor causes to be near the stability limits, or near the marginal stability, the parameter $k_{31}$ is determinant to the dynamics of the system, and the magnitude of the effect is big.

2) When $I_{1}, I_{2}<I_{3}$, case 1 , and the stability is such that the stability limit is in the proximity, negative values of $k_{31}$ contribute to system instability, and positive values contribute to stability. If the stability limit is not in the proximity, the effect of $k_{31}$ is negligible.

3) When $I_{3}<I_{1}, I_{2}$, negative values of $k_{31}$ contribute to stability if the stability limit is in the proximity. If the stability limit is not in the proximity, the effect of $k_{31}$ is negligible.

4) When rotation takes place around the intermediate inertia axis, $I_{1}<I_{3}<I_{2}$ or $I_{2}<I_{3}<I_{1}$, case 3 or 4 , it is difficult to obtain a practical stable configuration. However, if $k_{31}$ is big enough, it is possible to obtain an asymptotically stable configuration. In other words, the aerodynamic forces acting on the blades contribute to stability when spinning is close to the intermediate inertia axis, and the gravity forces contribute when the mass center is below the plane of blades.

The parameters $\left(N_{e}^{\prime}, k_{e}\right)$ can be considered to predict the stability behavior of a specific case by analyzing the stability regions presented. The characterization of a pararotor in terms of these parameters, which includes the main physical characteristics,

Table 5 Principal moments of inertia and weights for tested models

\begin{tabular}{ccccc}
\hline \hline$r_{31}, \mathrm{~m}$ & Weight, $\mathrm{N}$ & $I_{1}, \mathrm{~kg} \cdot \mathrm{m}^{2}$ & $I_{2}, \mathrm{~kg} \cdot \mathrm{m}^{2}$ & $I_{3}, \mathrm{~kg} \cdot \mathrm{m}^{2}$ \\
\hline 0.00 & 3.5 & $4.61 \cdot 10^{-4}$ & $1.31 \cdot 10^{-3}$ & $1.48 \cdot 10^{-3}$ \\
0.20 & 4.2 & $5.30 \cdot 10^{-4}$ & $1.56 \cdot 10^{-3}$ & $1.66 \cdot 10^{-3}$ \\
-0.20 & 4.2 & $5.30 \cdot 10^{-4}$ & $1.56 \cdot 10^{-3}$ & $1.66 \cdot 10^{-3}$ \\
\hline \hline
\end{tabular}

Table 6 Parameters measured and laboratory instruments used

\begin{tabular}{lc}
\hline \hline Parameter & Instrument \\
\hline Blade pitch $\beta_{1}, \beta_{2}$ & Goniometer \\
Stream velocity $U_{\infty}$ & Standard pitot tube and \\
& micromanometer \\
Angular velocity $\omega_{3}$ & Laser tachometer \\
Traction $F$ & Load cell \\
Nutation angle $\theta$ & Photo camera \\
Dry bulb ambient temperature & Dry and wet bulb thermometer \\
$T_{\text {amb }}$ & \\
Barometric pressure $P_{\text {amb }}$ & Electronic barometer \\
\hline \hline
\end{tabular}

Table 7 Experimental results and stability parameters

\begin{tabular}{|c|c|c|c|c|c|c|}
\hline \multicolumn{5}{|c|}{ Experimental results } & \multicolumn{2}{|c|}{$\begin{array}{c}\text { Stability } \\
\text { parameters }\end{array}$} \\
\hline$k_{31}$ & $\beta_{1}, \operatorname{deg}$ & $\beta_{2}, \operatorname{deg}$ & $U_{\infty}, \mathrm{m} / \mathrm{s}$ & $\omega_{3}, \mathrm{rad} / \mathrm{s}$ & $N_{e}^{\prime}$ & $k_{e}$ \\
\hline 0.0 & 4 & 4 & 9.5 & 235.2 & 0.080 & 9.724 \\
\hline 0.0 & 4 & 4 & 11.6 & 271.3 & 0.080 & 9.724 \\
\hline 0.0 & 4 & 8 & 10.1 & 255.9 & 0.080 & 9.724 \\
\hline 0.0 & 4 & 8 & 11.9 & 287.4 & 0.080 & 9.724 \\
\hline 0.0 & 4 & 12 & 9.7 & 268.4 & 0.080 & 9.724 \\
\hline 0.0 & 4 & 12 & 11.8 & 338.6 & 0.080 & 9.724 \\
\hline 0.2 & 4 & 4 & 10.2 & 228.9 & 0.070 & 18.987 \\
\hline 0.2 & 4 & 4 & 12.1 & 364.8 & 0.088 & 18.987 \\
\hline 0.2 & 4 & 8 & 10.1 & 261.9 & 0.082 & 18.987 \\
\hline 0.2 & 4 & 8 & 12.1 & 307.5 & 0.081 & 18.987 \\
\hline 0.2 & 4 & 12 & 10.1 & 269.3 & 0.086 & 18.987 \\
\hline 0.2 & 4 & 12 & 12.1 & 321.7 & 0.086 & 18.987 \\
\hline-0.2 & 4 & 4 & 10.0 & 201.0 & 0.210 & 18.987 \\
\hline-0.2 & 4 & 4 & 12.0 & 240.0 & 0.211 & 18.987 \\
\hline-0.2 & 4 & 8 & 9.9 & 228.2 & 0.198 & 18.987 \\
\hline-0.2 & 4 & 8 & 12.0 & 271.6 & 0.199 & 18.987 \\
\hline-0.2 & 4 & 12 & 10.1 & 246.1 & 0.192 & 18.987 \\
\hline-0.2 & 4 & 12 & 12.1 & 286.6 & 0.193 & 18.987 \\
\hline
\end{tabular}


configures a model to study the dynamic behavior of pararotor. Taking into account the relationship of principal moments of inertia and analyzing the effect of $k_{31}$ on stability, the following can be seen.

1) When $I_{1}, I_{2}<I_{3}$ or $I_{3}<I_{1}, I_{2}$, the stable region is given by the condition $N_{e}^{\prime}<1$. The first and second quadrants of the plane $\left(N_{e}^{\prime}, k_{e}\right)$ are allowed. Positive values of $k_{31}$ (that means that the center of mass is under the plain of the blades) makes $N_{e}^{\prime}$ smaller, reinforcing the tendency to asymptotic stability.

2) When $I_{1}<I_{3}<I_{2}$ or $I_{2}<I_{3}<I_{3}$, the stable region is given by $N_{e}^{t}>1$. The third and fourth quadrants of the plane $\left(N_{e}^{t}, k_{e}\right)$ are allowed.

Figure 5 and Table 4 show the behavior of system stability in different numerical examples, taking into account the eigenvalues of the characteristic matrix of the linearized system dynamics. It can be noticed that, near the stability limits, the effect of $k_{31}$ is determinant (Fig. 5, case 1.b), whereas for the rest of the cases, it has minor effect, even if the module of $k_{31}$ is on the order of 1 .

In the same way as in [15], it is possible to determine the nutation angle $\theta$, the precession angle $\psi$, and the spin angle $\varphi$ by decomposing the angular velocities given by $\omega$ over the mobile coordinate system. As the equilibrium point found is largely dependent on $k_{31}$, so are the Euler angles.

The results shows that induced velocity would affect the $N_{e}^{\prime}$ parameter as it should be added to the descent velocity present in the parameter $k$. However, induced velocity does not take effect neither on the stability characterization nor in the behavior of the system under the effect of the studied parameters change.

The experimental results validates, in the range defined by the tested models, the stability prediction derived from the mathematical model respect to the effect of $k_{31}$ on the stability.

\section{Discussion}

The way pararotors fly, the response to external disturbances, or the trajectories around different equilibrium points depend on a limited number of parameters whose effects are intrinsically coupled. Among them, the main influence comes from the relationships between moments of inertia.

The linear behavior characterization allows the dynamics of pararotors to be predicted or a preliminary design to be started by establishing functional flight and control requirements. Also, it is possible to establish control actions to guide the pararotor. This work, together with the existing references, represents the initial step to the development of technologically feasible pararotor missions.

The linear analysis is valid in all of the parameter range (if small angle assumption is met), except close to the stability limits, where second-order terms become relevant. Therefore, the main limitation of the present work is in the prediction accuracy close to the stability limits.

A pure analytical model that comprises the whole nonlinear system would be very complex and impractical. A numerical simulation tool is being developed with this aim.

\section{Conclusions}

The influence of parameters in the equilibrium attitude and dynamics of a pararotor has been analyzed by using a mathematical model based on the Euler equations and an expansion of the aerodynamic torque.

Considering different configurations and physical parameters of a pararotor, the most important for the dynamic stability and attitude has resulted to be the relationship between the principal moments of inertia $I_{1}, I_{2}$, and $I_{3}$. In practical terms, only devices where $I_{1}, I_{2}<I_{3}$ or $I_{3}<I_{1}, I_{2}$ lead to feasible configurations.

In general, the importance of the effect of distance from the blade plane to the center of mass $k_{31}$ depends on the magnitude of this parameter. However, the effect of this parameter depends also on the proximity of the system stability to the stability limits, being determinant when the system is close to marginal stability. For all cases, $k_{31}$ affects the equilibrium position of the pararotor, in terms of Euler angles.
The method considered to analyze the pararotor dynamics is based on the linearization of a reduced characteristic matrix of the Euler equations. The nonlinearities of the system become relevant to determine the character of the evolution when the system is approaching the stability limit. Thus, except in a region very close to the stability limit, the results obtained with the analysis presented are considered valid.

However, to study this region, a numerical integration of the complete model (without approximations) should be carried out, thus including nonlinear effects. The analytical study including nonlinear terms has been tried, but the large number of them that should be taken into account spoils the advantages of the analytical methods. A numerical model for the integration of the equations of the model is being developed by the authors. The analytical model is giving an unvaluable support in the validation of the model.

The parameter $k_{31}$ and the pitch angle could be used to control the response of the system, as can be determined from the linearization process of the dynamic system. Both are technologically feasible and affect the stability conditions of the system and the equilibrium points.

\section{References}

[1] Shpund, Z., and Levin, D., "Measurement of the Static and Dynamic Coefficients of a Cross-Type Parachute in Subsonic Flow," 11th AIAA Aerodynamic Decelerator Systems Technology Conference, AIAA Paper 1991-0871, April 1991.

[2] Levin, D., and Shpund, Z., "Dynamic Investigation of the Angular Motion of a Rotating Body-Parachute System," Journal of Aircraft, Vol. 32, No. 1, 1995, pp. 93-99. doi: $10.2514 / 3.46688$

[3] Shpund, Z., and Levin, D., "Forebody Influence on Rotating Parachute Aerodynamic Properties," Joumal of Aircraft, Vol. 34, No. 2, 1997, pp. 181-186. doi: $10.2514 / 2.2170$

[4] Levin, D., and Shpund, Z., "Canopy Geometry Effect on the Aerodynamic Behaviour of Cross-Type Parachutes," Journal of Aircraft, Vol. 34, No. 5, 1997, pp. 648-652. doi: $10.2514 / 2.2224$

[5] Karlsen, L., Borgström, D., and Paulsson, L., "Aerodynamics of a Rotating Body Descending from the Separation Position of an Artillery Munition Shell," 11th AIAA Aerodynamic Decelerator Systems Technology Conference, AIAA Paper 1991-0870, April 1991.

[6] Rosen, A., and Seter, D., "Vertical Autorotation of a Single-Winged Samara," Journal of Applied Mechanics, Vol. 58, No. 4, Dec. 1991, pp. 1064-1071. doi:10.1115/1.2897683

[7] Seter, D., and Rosen, A., "Stability of the Vertical Autorotation of a Single Winged Samara," Journal of Applied Mechanics, Vol. 59, No. 4, Dec. 1992, pp. 1000-1008.

doi:10.1115/1.2894014

[8] Crimi, P., "Analysis of Samara-Wing Decelerator Steady-State Characteristics," Journal of Aircraft, Vol. 25, No. 1, 1988, pp. 41- 47. doi: $10.2514 / 3.45539$

[9] Piechocki, J., "Estudio de la Dinámica del Vuelo de un Decelerador Aerodinámico Basado en el Concepto de Pararotor," Ph.D. Thesis, National Univ. of La Plata, Buenos Aires, Argentina, March 2011.

[10] Nadal Mora, V., "Comportamiento Aerodinámico de Sondas Atmosféricas en Entornos Aeroportuarios," Ph.D. Thesis, Technical Univ. of Madrid, Madrid, May 2005.

[11] Nadal Mora, V., Piechocki, J., and Pezzotti, S., "Ensayos en Túnel Aerodinámico de Modelos de Deceleradores Aerodinámicos de alas Rotatorias," Proceedings of the Congreso Argentino de Ingeniería Aeronáutica 2, Instituto Universitario Aeronáutico, Córdoba, Argentina, 2010.

[12] Piechocki, J., Nadal Mora, V., and Pezzotti, S., "Modelos Teóricos y Numéricos para Predecir el Comportamiento Dinámico de Pararrotores," Proceedings of the Congreso Argentino de Ingeniería Aeronáutica 2, Instituto Universitario Aeronáutico, Córdoba, Argentina, 2010.

[13] Piechocki, J., Nadal Mora, V., and Pezzotti, S., "Estudio Teórico del Efecto del Desplazamiento del Plano que Contiene a las Palas con Respecto al Centro de Masas en la Dinámica del Vuelo de un Desacelerador Aerodinámico de alas Rotatorias," Proceedings of the I Congreso Argentino de Ingeniería Mecánica, FoDAMI, Bahía Blanca, Argentina. 2008. 
[14] Sanz Andres, A., Nadal Mora, V., and Piechocki, J., "Pararotors for Planetary Atmosphere Exploration," Proceedings of the International Planetary Probe Workshop 5, Burdeos, France, 2007.

[15] Nadal Mora, V., Sanz Andrés, A., and Cuerva, A., "Model of the Aerodynamic Behaviour of a Pararotor," Journal of Aircraft, Vol. 43, No. 6, 2006, pp. 1893-1903. doi: $10.2514 / 1.21435$

[16] Nadal Mora, V., and Sanz, A., "Stability Analysis of a Free Falling Pararotor," Journal of Aircraft, Vol. 43, No. 4, 2006, pp. 980-986. doi:10.2514/1.16746

[17] Dorrington, G. E., "Concept Options for the Aerial Survey on Titan," Advances in Space Research, Vol. 47, No. 1, 2011, pp. 1-19. doi:10.1016/j.asr.2010.08.033

[18] Kellas, A., "The Guided Samara: Design and Development of a Controllable Single-Bladed Autorotating Vehicle," M.S. Thesis, Dept. of Aeronautics and Astronautics, Massachusetts Inst. of Technology, Cambridge, MA, 2007.

[19] Steiner, T. J., and Young, L. A., "Rotary Wing Decelerator Use on Titan," Proceedings of the International Planetary Probe Workshop 8, NASA, Portsmouth, EEUU, 2011
[20] Young, L. A., Briggs, G., Aiken, E., and Pisanich, G., "RotaryWing Decelerators for Probe Descent Through the Atmosphere of Venus," Defense Technical Information Center, Fort Belvoir, VA, 2004.

[21] Nadal Mora, V., Piechocki, J., Padfiel, G. D., and Sanz-Andres, A., "Experimental Research on a Vertically Falling Rotating Wing Decelerator Model," 19th ALAA Aerodynamic Decelerator Systems Technology Conference and Seminar, AIAA Paper 2007-2538, May 2007.

[22] Spedding, G. R., and McArthur, J., "Span Efficiencies of Wings at Low Reynolds Numbers," Journal of Aircraft, Vol. 47, No. 1, 2010, 120-128. doi:10.2514/1.44247

[23] Leishman, J. G., Principle of Helicopter Aerodynamics, Cambridge Aerospace Series, Cambridge Univ. Press, Cambridge, England, U.K., 2000, 36-76.

[24] Padfiel, G. D., Helicopter Flight Dynamics, Blackwell Science, Oxford, England, U.K., 1996, 25-53.

[25] Chi-Tsong, C., "Linear System Theory and Design," 3rd ed., Oxford Univ. Press, Oxford, England, U.K., 1999, 121-140. 